\title{
Instantaneous and cumulative influences of competition on impulsive choices in domestic chicks
}

\author{
Hidetoshi Amita ${ }^{1,2}$ and Toshiya Matsushima* ${ }^{*}$ \\ ' Graduate School of Life Science, Hokkaido University, Sapporo, Japan \\ 2 Japan Society for the Promotion of Science, Tokyo, Japan \\ ${ }^{3}$ Laboratory for Animal Behavior and Intelligence, Department of Biology, Faculty of Science, Hokkaido University, Sapporo, Japan
}

\section{Edited by:}

Marijn Van Wingerden, Heinrich-Heine

University Duesseldorf, Germany

\section{Reviewed by:}

Philippe N. Tobler, University of

Zurich, Switzerland

Timothy Cheung, Arizona State

University, USA

${ }^{*}$ Correspondence:

Toshiya Matsushima, Department of Biology, Faculty of Science, Hokkaido University, Sapporo 060-0810, Japan e-mail:matusima@sci.hokudai.ac.jp
This study examined instantaneous and cumulative effects of competitive interactions on impulsiveness in the inter-temporal choices in domestic chicks. Chicks were trained to peck colored beads to gain delayed food rewards ( 1 or 6 grains of millet delivered after a delay ranging between 0 and $4.5 \mathrm{~s}$ ), and were tested in binary choices between a small-short delay option (SS) and a large-long delay alternative $(\boldsymbol{L L})$. To examine whether competitive foraging instantaneously changes impulsiveness, we intraindividually compared choices between two consecutive tests in different contexts, one with competitors and another without. We found that (1) the number of the choice of $\boldsymbol{L} \boldsymbol{L}$ was not influenced by competition in the tests, but (2) the operant peck latency was shortened by competition, suggesting a socially enhanced incentive for food. To further examine the lasting changes, two groups of chicks were consecutively trained and tested daily for 2 weeks according to a "behavioral titration" procedure, one with competitors and another without. Inter-group comparisons of the choices revealed that (3) choice impulsiveness gradually decreased along development, while (4) the chicks trained in competition maintained a higher level of impulsiveness. These results suggest that competitive foraging causes impulsive choices not by direct/contextual modification. Causal link between the instantaneous enhancement of incentive and the gradual effects on impulsiveness remains to be examined. Some (yet unspecified) factors may be indirectly involved.

Keywords: inter-temporal choices, social facilitation, social foraging, work investment, foraging effort, operant conditioning, nucleus accumbens

\section{INTRODUCTION}

Social interferences could shift behaviors that maximize the individual payoff. When an animal is foraging in competition, a food item that is spatially/temporally remote should inevitably include a higher collection risk (McNamara and Houston, 1987; Benson and Stephens, 1996), and hence decision makers may reasonably redirect their choices toward a more proximate food item even though a large alternative is available. Such a rational forager could instantaneously change its impulsiveness as soon as a potential competitor appears; otherwise, it could change its choices much more slowly and gradually after accumulating the experiences of benefits and costs.

In fact, we found, by using domestic chicks as subjects, that competitive foraging enhances impulsive choices without actual interference of individual gains (Amita et al., 2010). In intertemporal choices between a small-short delay option $(S S)$ and a large-long delay alternative $(\boldsymbol{L} \boldsymbol{L})$, chicks that had been trained in competition for 3 days chose the $S S$ option significantly more frequently than those trained without competition. Chicks in both groups were tested in the same isolated condition, and the observed difference in impulsiveness was ascribed to the cumulative experiences of the perceived competition. It thus remained to be examined whether competitive foraging could cause instantaneous/contextual modification in choices and what (if any) of these modifications could underlie the observed cumulative effects.

Instantaneous effects of social facilitation have been documented in the work investments (running distance and pecking for food) in the accompanying paper by Ogura and Matsushima (2011). In the present study, on the other hand, we showed the other factors that may possibly be involved in the development of impulsiveness. First, we demonstrate that perceived competition instantaneously enhances the incentive for food, even though the choice of impulsiveness remains unchanged. The perceived competition shortened the operant peck latency, possibly as a form of social facilitation, without apparent increase in work investments. Second, we show that impulsiveness decreases by age/experience but the cumulative effects of competition are lasting, reconfirming the conclusion of our previous study (Amita et al., 2010).

\section{MATERIALS AND METHODS ANIMALS}

A total of 24 male chicks (Gallus domesticus, White Leghorns) were trained, but 3 chicks were discarded because they emitted distress calls and did not eat the millet food in the operant chamber. The present study is thus based on data obtained from 21 successfully trained individuals. In addition, 18 chicks served as companion individuals, but their behaviors were not recorded. New hatchlings 
(post-hatch day 1) were purchased from a local supplier, and housed in transparent plastic cages $(15 \mathrm{~cm} \times 28 \mathrm{~cm} \times 12 \mathrm{~cm})$ that were thermo-controlled at ca. $30^{\circ} \mathrm{C}$ under illumination (12L:12D, light period starting at 08:00). Each cage contained three chicks, all of which were trained and tested in the same conditions. On post-hatch day 2-4, each chick was fed with 1-3 g of food per day (mixture of millet and chick mash food). On day 5 and afterward, each chick received $0.5-1.0 \mathrm{~g}$ of millet during the experiments and was then fed once with $4 \mathrm{~g}$ rations in the evening. Water was freely available. After the end of the experiments, the chicks were sacrificed with carbon dioxide. Experiments were conducted under the guidelines and with the approval of the Committee on Animal Experiments of Hokkaido University. The guidelines are based on the national regulations for animal welfare in Japan (Law for the Humane Treatment and Management of Animals; after a partial amendment No. 68, 2005).

\section{APPARATUS}

We used an operant chamber for recording behaviors in the inter-temporal choice paradigm. A thermo-controlled box $\left(21 \mathrm{~cm} \times 19 \mathrm{~cm} \times 25 \mathrm{~cm}\right.$, maintained at ca. $27-30^{\circ} \mathrm{C}$ and illuminated by light bulbs) was used (see Aoki et al., 2006). One of the surrounding walls was equipped with a pair of holes placed side by side (separated by $3 \mathrm{~cm}$ and placed $4 \mathrm{~cm}$ above floor level), through which one or two colored beads (green, blue, or red) were presented for $1 \mathrm{~s}$. When a chick pecked at a bead associated with a reward, the millet food was supplied to the central food tray on the floor (placed between the two holes) after a programmed delay. Colored beads were assigned to reward options: small-short delay food ( $S S$ delivered after a constant mechanical lag $\Delta=0.29 \mathrm{~s}$ in average) and large-long delay food ( $\boldsymbol{L} \boldsymbol{L}$ delivered after delay $+\Delta)$. We observed the behaviors of the chicks through a video camera placed above the feeder, without being seen by the subject chicks. In experiment 1 , the chamber was divided into two sections by a transparent Plexiglas partition. A subject was trained and tested in one section, and a pair of free-riding companion chicks received food in the opposite section (Figure 1A). Each section was equipped with a feeder, and the two feeders were separated by $3 \mathrm{~cm}$. The chicks could see each other through the Plexiglas partition; the beads and food trays were also visible. As described previously (Amita et al., 2010), the fictitious social foraging that is not accompanied by actual interference of individual gain is referred to as "perceived competition."

\section{PROCEDURES}

\section{Experiment 1}

A blue bead was associated with a large-long delay reward $(\boldsymbol{L} \boldsymbol{L}, 2$ grains delivered after a long delay $=1.5 \mathrm{~s}+\Delta)$, and a red bead was associated with a small-short delay alternative $(S S, 1 / 3$ grain after $\Delta)$. A green bead was non-rewarding $(S-)$. Chicks were trained in two blocks (a no-competition and a competition block) per day for three successive days (post-hatch day 7-9), and subsequently tested in two blocks (a no-competition and a competition block) on day 10 (Figure 1A). The order of the no-competition and the competition blocks was randomized in both training and test. The training block consisted of 48 pseudo-randomly arranged trials: 12 trials with $L L / S-, 12$ trials with $S S / S-$, and 24 trials with $S-/ S-$.

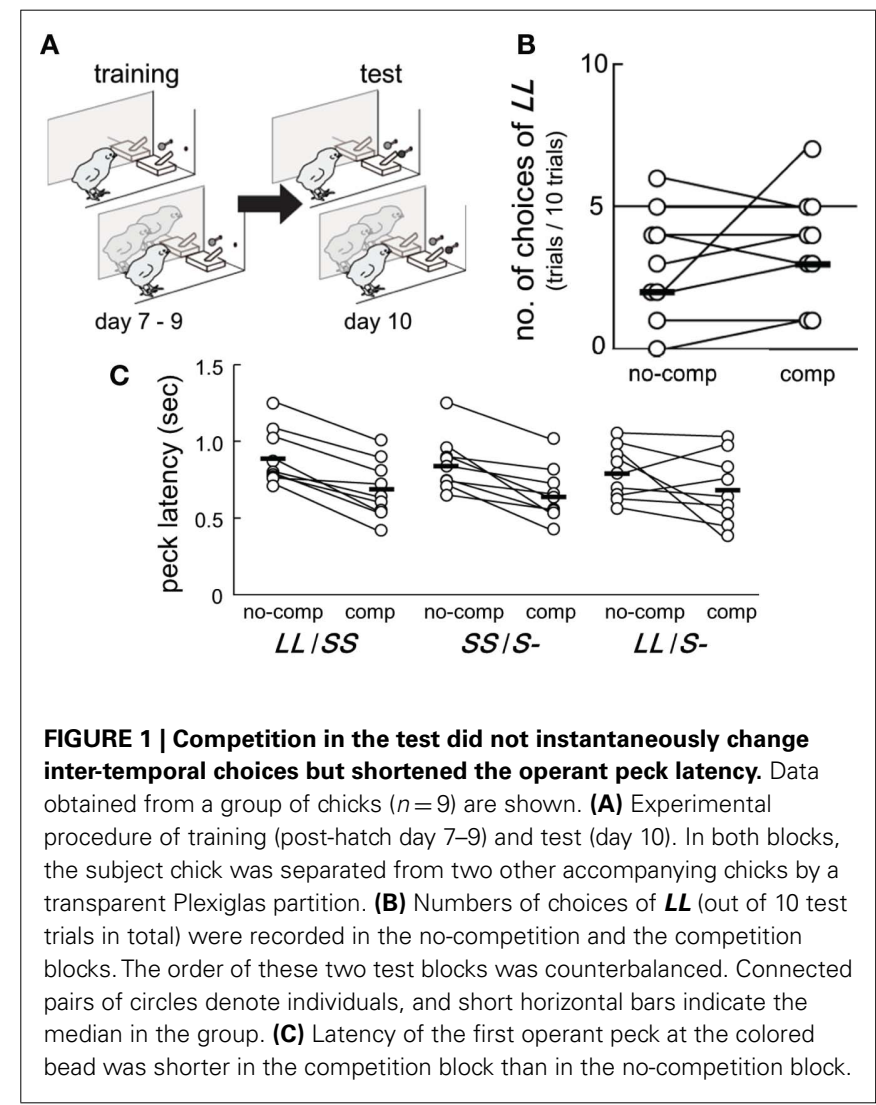

The test block consisted of 60 pseudo-randomly arranged trials: 10 trials with $L L / S S, 10$ trials with $L L / S-, 10$ trials with $S S / S-$, and 30 trials with $S-/ S-$. Inter-trial intervals varied between 15 and $20 \mathrm{~s}$. ITIs were not adjusted according to the trial types. In order to mimic the variation in the food gain in the group of three chicks, we assumed that each chick had an equal chance to get each grain in the competition and set the amount to vary at every trial according to a binomial distribution (see Amita et al., 2010). For $S S, 1$ grain was supplied in 24 trials and no food was supplied in the remaining 48 trials so that the mean was $1 / 3$ grain per trial for 72 trials ( 0 grain in 48 trials and 1 grain in 24 trials; pseudorandomly arranged sequence). Similarly, for $\boldsymbol{L} \boldsymbol{L}, 0-6$ grains were supplied and the mean amount was set at 2 grains per trial for 72 trials ( 0 grain in 11 trials, 1 grain in 12 trials, 2 grains in 26 trials, 3 grains in 16 trials, 4 grains in 4 trials, 5 grains in 2 trials and 6 grains in 1 trial; pseudo-randomly arranged sequence). On day 10 (test), 1 and 6 grains were given for $S S$ and $\boldsymbol{L} \boldsymbol{L}$, respectively. The pair of companion chicks was given 3 grains at the time when the subject gained food.

\section{Experiment 2}

A blue bead was associated with a large-long delay reward ( $\boldsymbol{L} \boldsymbol{L}, 6$ grains delivered after a long delay), and a red bead was associated with a small-short delay alternative ( $S S, 1$ grain after a delay of $\Delta)$. A green bead was non-rewarding $(S-)$. Chicks were trained for 12 days, from post-hatch day 7 to 19 (except day 13), either in isolation (no-competition) or in a group of 3 individuals (competition; Figure 2A). Note that experiment 2 is a between-subject 

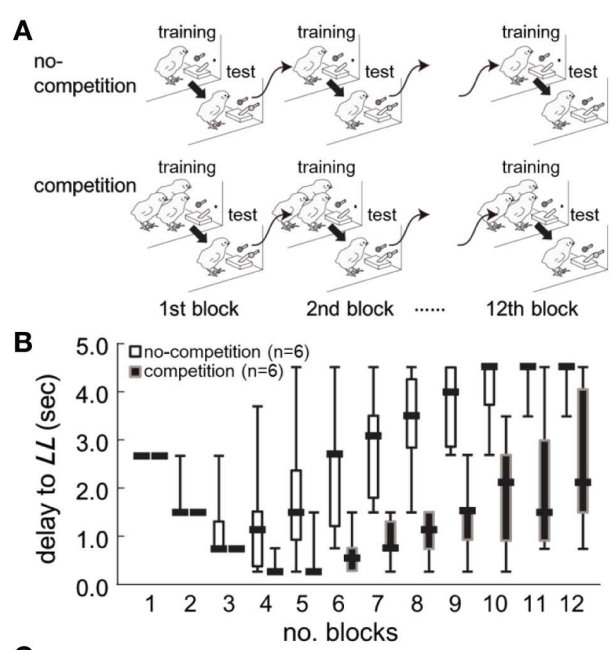

C

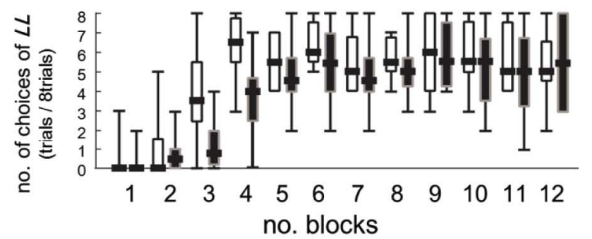

FIGURE 2 | Competition caused lasting and cumulative effects on impulsiveness. Data obtained from the two groups of chicks ( $n=6$ each) are shown. (A) Experimental procedure of the behavioral titration. By adjusting the delay to the large-long delay reward [ $\boldsymbol{L} \boldsymbol{L}, \mathbf{B})]$, we searched for an equilibrium point at which both options were equally chosen (C). Chicks were trained and tested for 12 days (1 block per day) from post-hatch day 7 . In the no-competition group (upper), chicks were trained in isolation. In the competition group (lower), chicks were trained in a group of three chicks. Note that chicks were tested in isolation in both groups. (B) Delay to $\mathbf{L} \boldsymbol{L}$ was plotted against the number of blocks. Short horizontal bars indicate the median, boxes the $25-75 \%$ range, and whiskers the min-max range, respectively. (C) The number of choices of $\boldsymbol{L} \boldsymbol{L}$ was similarly plotted.

design. In competition, two chicks served as companion individuals. The partitioning Plexiglas was not used in experiment 2. It is noteworthy that the food was shared among individuals and the chick that pecked the bead did not necessarily gain all of the grain. Though we did not record the amount of food that each individual gained, we assumed that a longer delay to $\boldsymbol{L} \boldsymbol{L}$ did not lead to a higher probability of interception of the food by the companion individuals, because the interception occurred after the end of the delay period.

It might be argued that the competitor chicks interfere with the subject in learning in forming the association between the colored beads with rewards. The chicks in competition could learn the association more slowly than those in no-competition due to the distractive effects of competitors. In this study, in order to avoid the possible interference of learning, we gave pre-training blocks before the behavioral titration started (see below for details). We also accomplished a supplementary experiment (see Figure A2 in Appendix) in order to directly examine the effects of interference.

Before the titration procedure started, in order to make subject chicks to form the associations between the colored bead and the rewards, the chicks received two blocks of pre-training, one per day (day 5-6), either in isolation (no-competition) or in a group of three individuals (competition). One bead was presented per trial (namely, forced choice trials), and no binary choice trials were given in the pre-training. In pre-training blocks, the delay to $L L$ was set at 0.29 s. If the chicks pecked a green bead $(S-)$, the bead was repeatedly presented (up to five trials) until chick stayed not to peck the bead (correction trials). The pre-training block consisted of 72 pseudo-randomly arranged trials: 18 trials with $\boldsymbol{L} \boldsymbol{L}, 18$ trials with $\boldsymbol{S S}$ and 36 trials with $\boldsymbol{S}$-. The chicks received one block of training and one block of testing each day. The same chicks were used across test blocks. The training block consisted of 72 pseudo-randomly arranged trials: 18 trials with $\boldsymbol{L L} / \boldsymbol{S}_{-}, 18$ trials with $S S / S_{-}$, and 36 trials with $S-/ S_{-}$. The test block consisted of 48 pseudo-randomly arranged trials: 8 trials with $L L / S S$, 8 trials with $L L / S-, 8$ trials with $S S / S-$, and 24 trials with $S-/ S-$. Inter-trial intervals varied between 15 and $20 \mathrm{~s}$. For behavioral titration, we adopted a procedure similar to that employed by Kawamori and Matsushima (2010). Briefly, the delay to $\boldsymbol{L} \boldsymbol{L}$ was incremented (or decremented) in the $n$th training and testing block if the chick chose $\boldsymbol{L} \boldsymbol{L}$ for more than 5 (or less than 3 ) out of the $8 L L / S S$ test trials in the preceding $(n-1)$ th block. The delay to $\boldsymbol{L} \boldsymbol{L}$ was unchanged in the $n$th block if the chick chose $\boldsymbol{L} \boldsymbol{L}$ for 3-5 trials out of the 8 test trials in the $(n-1)$ th block. The choice ratio was adjusted by changing the $\boldsymbol{L} \boldsymbol{L}$ delay in six steps $(0.29,0.75,1.50,2.66,3.48$, and $4.51 \mathrm{~s}$, including the mechanical lag $\Delta=0.29 \mathrm{~s})$.

We daily measured the subjects' body weight during behavioral titration but detected no differences between the groups (see Figure A3 and Table A5 in Appendix).

\section{STATISTICAL ANALYSIS}

We analyzed data by using $\mathrm{R}$ (computer language developed for statistical computations, version 2.6.0). Generalized linear mixed models (GLMMs) were constructed to fit the observed data: the number of choices and the peck latency in experiment 1 (Tables A1 and $\mathbf{A} 2$ in Appendix), and the delay to $L L$ in experiment 2 (Table A4 in Appendix). For the number of choices in the test phase of experiment 2, multiple comparisons by a binomial test were used after sequential Bonferroni corrections (Table A3 in Appendix).

\section{RESULTS \\ EXPERIMENT 1}

Perceived competition did not cause any instantaneous changes in inter-temporal choices, but clearly shortened the operant peck latency at test (day 10). Results showed that six out of nine chicks chose $\boldsymbol{L} \boldsymbol{L}$ less frequently than $S S$ in both the no-competition and the competition blocks (Figure 1B). The numbers of choices of $\boldsymbol{L} \boldsymbol{L}$ were fitted by GLMM (Table A1 in Appendix) by including competition (no-competition or competition block) and order (whether the competition block preceded or followed by the no-competition block) as explanatory variables. Model selection by Akaike information criteria (AIC) showed that the null model yielded a smaller AIC $(=30.74)$ than the competition model (31.76), the order model (32.74), and the competition + order model (33.76). Therefore, we are unable to conclude that perceived competition instantaneously changes the choices. 
In all nine chicks, peck latency was shorter in the competition block than in the no-competition block in both the $L L / S S$ and the $S S / S$ - trials (Figure 1C). Similarly, in the $L L / S$ - trials, seven of nine chicks showed shorter peck latency in the competition block. Peck latencies were fitted by GLMM (Table A2 in Appendix) by including competition, trial types $(S S / S-$ or $\mathbf{L L} / \boldsymbol{S}$ - and interactions between competition and trial types as explanatory variables. The model composed only of competition yielded the smallest AIC (=88.53), suggesting that competition shortened the peck latency irrespective of the trial types. However, in model 2, which had the second smallest AIC (=95.76), the estimated coefficient of the trial types was positive, suggesting that the latency might be longer in the $S S / S$ - trials than the $\boldsymbol{L} L / S$ - trials, somehow incompatible with the choice data (Figure 1B; see Discussion below). Furthermore, in model 3, which had a larger AIC, the estimated coefficient of the interaction term (between competition and trial types) was negative, suggesting that the effect of competition might be larger in the $S S / S$ - trials than in the $\boldsymbol{L} \boldsymbol{L} / \boldsymbol{S}$ - trials. We compared these four models (including the null model) by likelihood-ratio test, and found no significant differences between model 1 and $2(p=0.79)$ and also between model 1 and $3(p=0.34)$, but difference was significant between model 1 and $4(p<0.01)$. We therefore conclude that competition primarily contributed to the shortening latency.

\section{EXPERIMENT 2}

Competition caused lasting and cumulative effects on impulsiveness. The delay to $\boldsymbol{L} \boldsymbol{L}$ (Figure $\mathbf{2 B}$ ) served as a measure of the impulsiveness when the choices of $\boldsymbol{L} \boldsymbol{L}$ (Figure 2C) were balanced in the titration procedure. We compared the choice data in each block by using a binomial test (Table A3 in Appendix), but the difference between the groups was significant only in the fourth block (sequential Bonferroni correction for multiple comparisons, Hommel, $1988 ; p<0.05)$. We therefore fitted the delay to $\boldsymbol{L} \boldsymbol{L}$ data from the 5th to 12th blocks by GLMM (Table A4 in Appendix) by including competition (no-competition or competition) and block (the number of the block) as explanatory variables (Table A4 in Appendix). The competition + block model yielded the smallest AIC (=42.74), whereas the block model (52.48) and the competition model (60.94) yielded larger AICs. The estimated coefficient of the block term was positive, indicating that in both groups, the impulsiveness decreased as the chicks grew. On the other hand, the estimated coefficient of the competition term was negative, indicating that competition caused a lasting facilitation on impulsiveness.

Competition may have interfered with the association between cues and rewards. In experiment 2, slower shift for $\boldsymbol{L} \boldsymbol{L}$ in the competition group may be explained by a slower learning because competitors could serve as distractors, which interfered with the associative learning. However, the chicks in both groups always chose $\boldsymbol{S}+$ beads in the $\boldsymbol{S}+/ \boldsymbol{S}$ - trials in the test blocks (Figure A1 in Appendix); the percentage of correct responses (no peck) did not differ also in the $S-/ S-$ trials. We therefore conclude that the effects of competition on impulsiveness cannot be ascribed to the interference in learning. Further direct examination (Figure A2 in Appendix) failed to reveal the possible interference by competition. If competition interfered with associative learning, the chicks would choose more frequently the colored bead learned in no-competition than that in competition. However, biased choices were not found between such color cues.

\section{DISCUSSION}

Perceived competition shortened the peck latency, but it failed to change instantaneously the impulsive choices (experiment 1). If the shorter response latency represented a more valuable option, as has been argued (Brown and Bowman, 1995; Lauwereyns and Wisnewski, 2006), the present results suggest that perceived competition enhances the chicks' incentive for food. The peck latency in the $S S / S$ - trials were, however, even longer than the $L \boldsymbol{L} / \boldsymbol{S}$ trials (Figure 1C), whereas the choices were biased in favor of the $S S$ (Figure 1B). It should be noted that the chicks always pecked the rewarding bead when the alternative was $S$ - (see Figure A1 in Appendix). We also found no significant correlation between the number of choice of $\boldsymbol{L} \boldsymbol{L}$ and the peck latencies at the individual level (data not shown). Therefore, shorter peck latency did not necessarily represent a more valuable option in this study using chicks.

It is possible that foraging incentive could have a direct control on the impulsiveness. It has been reported that a decrease in water deprivation level causes an increase in response latency in rats (Richards et al., 1997); they further examined the effects of the deprivation on impulsiveness, but found no effects. In our present study (experiment 1), similarly, perceived competition shorted the peck latency, but did not enhance the impulsiveness. We therefore argue that the incentive does not have a direct and strong link to the impulsive choices.

When viewed ecologically, the shortened peck latency could be an adaptive trait. For foragers that forage in kleptoparasitism, as do chicks, both forms of impulsiveness (in the pecking action and the choice of SS) might be adaptive traits. Particularly, in the framework of scramble kleptoparasitism, several individuals simultaneously exploit a food resource with little or no aggression and the players' payoff is assumed by the producer-scrounger game (Giraldeau and Caraco, 2000). Producers search their environment for food clumps, and scroungers attend to other foragers' discoveries and scrounge. The critical point is that the payoff depends on the proximity; the producer gains more from a more proximate food resource, and the scroungers gain more from more proximate producers (Di Bitetti and Janson, 2001). The shortened peck latency (experiment 1) and impulsive choices (experiment 2) could efficiently increase the forager's gain, in both the producer and the scrounger.

In the experimental situation adopted in this study, however, the impulsive choice did not contribute to a higher gain. The total amount of food decreased when chicks made more impulsive choices. Similarly, the shortened peck latency did not lead to a greater payoff, because the food amount remained unchanged irrespective of whether the chick rushed or not. These results suggest that the behaviors of chicks are predisposed and not dependent on the actual benefit that the subjects gain. Ecological accounts are also useful in this context. For foragers that find and eat tiny food particles, such as star-nosed mole rats, short handling time has a marked effect on profitability (energy gained per handling time; Catania and Remple, 2005). In chicks, similarly, even a slight shortening in the peck latency by a few tenths of a second could significantly increase the food profitability in the natural context of competitive foraging.

It remains to be elucidated how impulsiveness cumulatively changes after competitive experiences. As shown in the accompanying paper by Ogura and Matsushima (2011), the perception 
of other individuals is also effective in instantaneously increasing the work investments/foraging efforts. A similar competition, however, fails to cause an immediate change in impulsiveness, as revealed in this study. The shortened peck latency might be assumed as a form of social facilitation, as has been widely reported in psychology (Matlin and Zajonc, 1968). However, apparent increase in running distance and number of pecks were not found in this study. It is interesting to study whether the social facilitation without work investments/foraging efforts could cause the cumulative development of impulsiveness, and if so, which of the facilitated behaviors could be specifically responsible.

It is an open question as to whether the social facilitation and the impulsiveness have common or distinct neural mechanisms. Lesions of nucleus accumbens (NAc) induce impulsive choices in rats (Cardinal et al., 2001) and also in chicks (Izawa et al., 2003). Neurophysiological characterization of their NAc and the surrounding areas in the ventral striatum has revealed a population of neurons that selectively code the proximity and amount of anticipated food reward (Yanagihara et al., 2001; Izawa et al., 2005). A recent study in rats has also revealed that neurons in the ventral striatum represent the delay and the size of the food reward, and the firing rate was negatively correlated with the response latency (Roesch et al., 2009). It is plausible that the neural codes of reward anticipation in the ventral striatum could become modified after competition.

\section{REFERENCES}

Amalric, M., and Koob, G. F. (1987). Depletion of dopamine in the caudate nucleus but not in nucleus accumbens impairs reaction-time performance in rats. J. Neurosci. 7, 2129-2134.

Amita, H., Kawamori, A., and Matsushima, T. (2010). Social influences of competition on impulsive choices in domestic chicks. Biol. Lett. 6, 183-186.

Aoki, N., Suzuki, R., Izawa, E.-I., Csillag, A., and Matsushima, T. (2006). Localized lesions of ventral striatum, but not arcopallium, enhanced impulsiveness in choices based on anticipated spatial proximity of food rewards in domestic chicks. Behav. Brain Res. 168, 1-12.

Benson, K. E., and Stephens, D. W. (1996). Interruptions, tradeoffs, and temporal discounting. Am. Zool. 36, 506-517.

Brown, V. J., and Bowman, E. M. (1995). Discriminative cues indicating reward magnitude continue to determine reaction time of rats following lesions of the nucleus accumbens. Eur. J. Neurosci. 7, 2479-2485.

Cardinal, R. N., Pennicott, D. R., Sugathapala, C. L., Robbins, T. W., and Everitt, B. J. (2001). Impulsive choice induced in rats by lesions of the nucleus accumbens core. Science 292, 2499-2501.
Catania, K. C., and Remple, F. E. (2005). Asymptotic prey profitability drives star-nosed moles to the foraging speed limit. Nature 433, 519-522.

Day, J. J., Jones, J. L., Wightman, R. M., and Carelli, R. M. (2010). Phasic nucleus accumbens dopamine release encodes effort- and delayrelated costs. Biol. Psychiatry 68, 306-309.

Di Bitetti, M. S., and Janson, C. H. (2001). Social foraging and the finder's share in capuchin monkeys, Cebus apella. Anim. Behav. 62, 47-56.

Giraldeau, L.-A., and Caraco, T. (2000). Social Foraging Theory. Princeton, NJ: Princeton University Press.

Hommel, G. (1988). A stagewise rejective multiple test procedure based on a modified Bonferroni test. Biometrika 75, 383-386.

Izawa, E.-I., Aoki, N., and Matsushima, T. (2005). Neural correlates of the proximity and quantity of anticipated food rewards in the ventral striatum of domestic chicks. Eur. J. Neurosci. 22, 1502-1512.

Izawa, E.-I., Zachar, G., Yanagihara, S., and Matsushima, T. (2003). Localized lesion of caudal part of lobus parolfactorius caused impulsive choice in the domestic chick: evolutionarily conserved function of ventral striatum. J. Neurosci. 23, 1894-1902.

The neuromodulatory action of dopamine may accompany the instantaneous effects on the peck latency and the work investment. It is reported that low-cost cues produce a significantly larger increase in NAc dopamine concentration than do high-cost cues. Moreover, immediate reward cues in the delay task evoke a larger dopamine increase than do delayed reward cues (Day et al., 2010). On the other hand, experimental depletion of dopamine in the caudate nucleus increases response latency (Amalric and Koob, 1987). It is therefore possible that the enhanced release/action of dopamine in NAc could lead to the instantaneous effects of competition. Causal links between the instantaneous and the cumulative effects of competition need further experimental study.

\section{ACKNOWLEDGMENTS}

This study was supported by grant-in-aid for scientific research to Toshiya Matsushima from the Japanese Society for the Promotion of Sciences (JSPS \#22570070), as well as that for Innovative Area and from the Japanese Ministry of Education, Culture, Sports, Science, and Technology (MEXT; The study on the neural dynamics for understanding communication in terms of complex hetero systems; \#22120502). Fellowship and grant-in-aid for scientific research from JSPS to Hidetoshi Amita (\#22002260) are also highly appreciated. We would like to express our gratitude to anonymous referees, who patiently gave us detailed, generous, and critical comments on our initial manuscript.

Kawamori, A., and Matsushima, T. (2010). Subjective value of risky foods for individual domestic chicks: a hierarchical Bayesian model. Anim. Cogn. 13, 431-441.

Lauwereyns, J., and Wisnewski, R. G. (2006). A reaction-time paradigm to measure reward-oriented bias in rats. J. Exp. Psychol. 32, 467-473.

Matlin, M. W., and Zajonc, R. B. (1968). Social facilitation of word associations. J. Pers. Soc. Psychol. 10, 455-460.

McNamara, J. M., and Houston, A. I. (1987). A general framework for understanding the effects of variability and interruptions on foraging behavior. Acta Biotheor. 36, 3-22.

Ogura, Y., and Matsushima, T. (2011). Social facilitation revisited: increase in foraging efforts and synchronization of running in domestic chicks. Front. Neurosci. 5:91. doi: 10.3389/fnins.2011. 00091

Richards, J. B., Mitchell, S. H., De Wit, H., and Seiden, L. S. (1997). Determination of discount functions in rats with an adjusting-amount procedure. J. Exp. Anal. Behav. 67, 353-366.

Roesch, M. R., Singh, T., Brown, P. L., Mullins, S. E., and Schoenbaum, G. (2009). Ventral striatal neurons encode the value of the chosen action in rats deciding between differently delayed or sized rewards. J. Neurosci. 29, 13365-13376.

Yanagihara, S., Izawa, E.-I., Koga, K., and Matsushima, T. (2001). Rewardrelated neuronal activities in basal ganglia of domestic chicks. Neuroreport 12, 1431-1435.

Conflict of Interest Statement: The authors declare that the research was conducted in the absence of any commercial or financial relationships that could be construed as a potential conflict of interest.

Received: 02 May 2011; accepted: 17 August 2011; published online: 16 September 2011.

Citation: Amita $H$ and Matsushima $T$ (2011) Instantaneous and cumulative influences of competition on impulsive choices in domestic chicks. Front. Neurosci. 5:101. doi: 10.3389/fnins.2011.00101

This article was submitted to Frontiers in Decision Neuroscience, a specialty of Frontiers in Neuroscience.

Copyright (c) 2011 Amita and Matsushima. This is an open-access article subject to a non-exclusive license between the authors and Frontiers Media $S A$, which permits use, distribution and reproduction in other forums, provided the original authors and source are credited and other Frontiers conditions are complied with. 


\section{APPENDIX}

We focused on the number of choices of $\boldsymbol{L} L$ as the response variable. Since chicks were tested in binary choices, we assumed a binominal distribution for the error structure of the data of choice ratio, and the choice probability $=Q(X)(\in[0,1])$ was approximated by logit link function such as,

$$
Q(X)=1 /(1+\exp (-\boldsymbol{X}))
$$

in which a predictor $X$ was linearly given as a weighed sum of explanatory variables.

$X=\beta_{0}+\beta_{1} *$ competition $+\beta_{2} *$ order $+r_{i}$

The competition denotes a category type variable: 0 for nocompetition and 1 for competition, respectively, and the order (variable $=1,2$ ) denotes whether the test in competitive context was accomplished before (order $=1$ ) or after (order $=2$ ) the test in non-competitive context; $r_{i}$ denotes the individual difference. Note that the model 1 included only the intercept and individual random difference. In the model 2 with a slightly larger AIC value, however, the $95 \%$ confidence range of the $\beta_{1}$ involved 0 , indicating that the competition did not account for the choice.

Trials in $S S / S$ - and $L L / S$ - trials were included, but the data in $S S / L L$ trials were omitted. We assumed a Poisson distribution for the error structure of the data of the peck latency considering that they were all non-negative values. $\boldsymbol{\Lambda}(\boldsymbol{X})(>0)$ was thus approximated by a Poisson function (log-link function) as,

$$
\boldsymbol{\Lambda}(\boldsymbol{X})=\exp (\boldsymbol{X})
$$

in which a predictor $X$ was linearly given as a weighed sum of explanatory variables.

$$
\begin{aligned}
X= & \beta_{0}+\beta_{1} * \text { competition }+\beta_{2} * \text { trail types } \\
& +\beta_{3} * \text { competition:trail types }+r_{i}
\end{aligned}
$$

The competition denotes a category type variable: 0 for nocompetition and 1 for competition, respectively. The trial types denotes a category type variable: 0 for $L L / S-$ and 1 for $S S / S-$. $r_{i}$ denotes the individual difference.
Estimated coefficients $\beta_{1}$ of the effects of competition in the models 1 to 3 suggest that the chicks shortened the peck latency in competitive block, irrespectively of the trial types. The model 1 that took only competition into account was chosen with the smallest AIC value. On the other hand, the model 2 suggests a positive $\beta_{2}$ coefficient for trial types, suggesting latency was slightly longer in the $S S / S$ - trials than $L L / S$ - trials. Similar tendency was detected in the model 3 , in which $\beta_{3}$ (for interaction between competition and trial type) was negative, suggesting that the competition effect was larger in the $S S / S$ - trials than $L \boldsymbol{L} / \boldsymbol{S}$ - trials.

Between the two groups of chicks (no-competition vs. competition), statistically significant differences were found only on day 4 , in which the calculated $p$-value was smaller than $\pi /(n-i+1)$. Here, the blocks were rearranged in the order of the $p$-value; $n$ denotes the total number of blocks (12), and $i$ the block number (1-12), respectively. The experiment-wide level of significance was set at $\pi=0.05$.

The model that took both competition and block number into account was chosen with the smallest AIC value. In constructing the model, we focused on the delay to $\boldsymbol{L} \boldsymbol{L}$ as the response variable. Since the delays were non-negative discrete values, we assumed a Poisson distribution for the error structure of the data. A delay to $L \boldsymbol{L}=\boldsymbol{Q}(\boldsymbol{X})(>0)$ was thus approximated via log-link function as

$Q(X)=\exp (X)$

in which the linear predictor $\boldsymbol{X}$ was given as a weighed sum of explanatory variables.

$\boldsymbol{X}=\beta_{0}+\beta_{1} *$ competition $+\beta_{2} *$ block $+r_{i}$

The competition denotes a category type variable: 0 for nocompetition and 1 for competition, and the block denotes a variable (=5-12), respectively. Coefficient $\beta_{1}$ indicates how the competition contributed, and $\beta_{2}$ indicates how the number of blocks contributed to the delay to $L \boldsymbol{L} ; \mathrm{r}_{\mathrm{i}}$ denotes the individual difference. Asterisks indicate that the $95 \%$ confidence range of the estimated coefficient did not involve 0 . The model 1 with model 2 were further compared by likelihood-ratio test, giving rise to a significant difference $(p=0.0006115)$. 
Table A1 | Numbers of choices of LL (data shown in Figure 1B) were analyzed by GLMM, and the null model that was composed of intercept ( $\beta_{0}$ ) and individual random difference $\left(r_{i}\right)$ was chosen.

\begin{tabular}{llll}
\hline Models & AIC & \multicolumn{2}{c}{ Estimated coefficients of variables } \\
\cline { 3 - 4 } & & $\beta_{\mathbf{0}}$ (intercept) & $\boldsymbol{\beta}_{\mathbf{1}}$ (competition) \\
\hline $1\left(\beta_{0}\right)$ & 30.74 & $-0.7647^{*}$ & - \\
$2\left(\beta_{0}, \beta_{1}\right)$ & 31.76 & $-0.9323^{*}$ & 0.3256 \\
$3\left(\beta_{0}, \beta_{2}\right)$ & 32.74 & -0.7646 & - \\
$4\left(\beta_{0}, \beta_{1}, \beta_{2}\right)$ & 33.76 & -0.8888 & 0.3284 \\
\hline
\end{tabular}

Table A2 | Peck latency (data shown in Figure 1C) was analyzed by generalized linear mixed model (GLMM) with log-link function.

\begin{tabular}{|c|c|c|c|c|c|}
\hline Models & AIC & \multicolumn{4}{|c|}{ Estimated coefficients of variables } \\
\hline $2\left(\beta_{0}, \beta_{1}, \beta_{2}\right)$ & 95.76 & 0.81146 & -0.14924 & 0.04902 & - \\
\hline $3\left(\beta_{0}, \beta_{1}, \beta_{2}, \beta_{3}\right)$ & 99.62 & 0.79102 & -0.14924 & 0.00732 & -0.08094 \\
\hline $4\left(\beta_{0}\right)$ & 108.1 & 0.73818 & - & - & - \\
\hline
\end{tabular}

Table A3 | Numbers of choices of $L L$ along the 12 test blocks (data shown in Figure $2 \mathrm{C}$ ) were analyzed by multiple comparisons after sequential Bonferroni corrections (Hommel, 1988).

\begin{tabular}{lllllllllllll}
\hline No. block & $\mathbf{4}$ & $\mathbf{3}$ & $\mathbf{6}$ & $\mathbf{1 0}$ & $\mathbf{5}$ & $\mathbf{7}$ & $\mathbf{8}$ & $\mathbf{2}$ & $\mathbf{1 1}$ & $\mathbf{1 2}$ & $\mathbf{1}$ & $\mathbf{9}$ \\
\hline$p$-Value & 0.0033 & 0.0049 & 0.2508 & 0.5089 & 0.5220 & 0.5220 & 0.6624 & 0.7576 & 0.8183 & 0.8286 & 1.0000 & 1.0000 \\
$\pi /(n-i+1)$ & 0.0042 & 0.0045 & 0.0050 & 0.0056 & 0.0063 & 0.0071 & 0.0083 & 0.0100 & 0.0125 & 0.0167 & 0.0250 & 0.0500 \\
\hline
\end{tabular}

Table A4 | Delay to LL (data shown in Figure 2B) was analyzed by generalized linear mixed model (GLMM).

\begin{tabular}{llll}
\hline Models & AIC & \multicolumn{2}{c}{ Estimated coefficients of variables } \\
\cline { 3 - 4 } & & $\beta_{\mathbf{0}}$ (intercept) & \multicolumn{1}{c}{$\boldsymbol{\beta}_{\mathbf{1}}$ (competition) } \\
\hline $1\left(\beta_{0}, \beta_{1}, \beta_{2}\right)$ & 42.74 & $0.6370^{*}$ & $-0.6118^{*}$ \\
$2\left(\beta_{0}, \beta_{2}\right)$ & 52.48 & 0.3278 & - \\
$3\left(\beta_{0}, \beta_{1}\right)$ & 60.94 & $1.5588^{*}$ & $-0.6119^{*}$ \\
$4\left(\beta_{0}\right)$ & 70.69 & $1.2496^{*}$ & - \\
\hline
\end{tabular}

Table A5 | Daily recorded body weight (data shown in Figure A3) was analyzed by generalized linear mixed model (GLMM) with a linear function. The models 1 and 2 yielded similar AIC values, but a comparison by likelihood-ratio test revealed no significant difference between the two models $(p=0.4492)$, suggesting that the competition did not influence the growth.




A

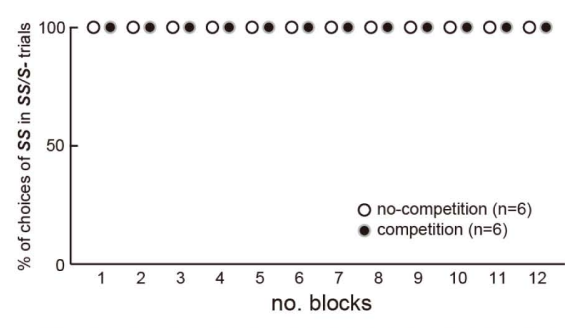

B

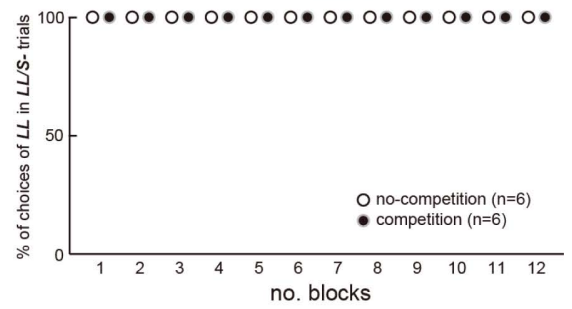

C

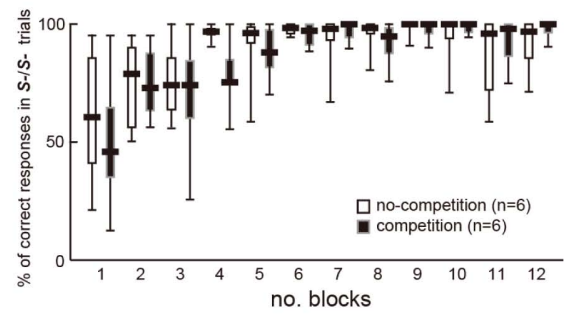

FIGURE A1 | No difference was found in their learning profiles between the two groups of chicks in experiment 2 ( $n=6$ each). (A) Percentage of choices of $\boldsymbol{S} \boldsymbol{S}$ in $8 \boldsymbol{S S} / \boldsymbol{S}$ - trials in each test block was plotted against the number of block. (B) Percentage of choices of $\boldsymbol{L} \boldsymbol{L}$ in $8 \mathbf{L} \boldsymbol{L} / \boldsymbol{S}$ - trials in each test block was plotted against the number of block. (C) Percentage of correct responses (no peck) to $\boldsymbol{S}_{-}$in $24 \boldsymbol{S}-\boldsymbol{S}_{-}$trials in each test block was plotted against the number of block. Short horizontal bars indicate the median, boxes $25-75 \%$ range, and whiskers min-max range, respectively. GLMM analysis of the \% correct responses in $\boldsymbol{S}-/ \boldsymbol{S}$ - trials revealed that the model with the smallest AIC included only the number of blocks as explanatory variable.
A

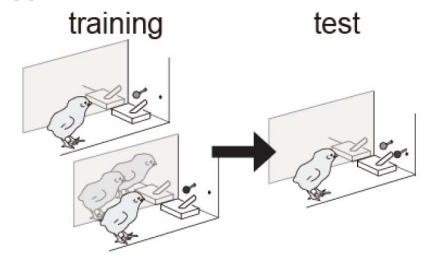

1st day $7-9$

day 10

2nd day $11-13$

day 14
B



FIGURE A2 | Biased choices were not found between the colored bead associated with competition and that with no-competition. Data obtained from a group of chicks $(n=6)$ are shown. (A) Experimental procedure of training and test. The subject chick was separated from the accompanying chicks by a transparent Plexiglas, so that actual interference of food reward did not occur. In the first set of blocks, a blue (or green) bead was associated with competition and 3 grains after $\Delta=0 \mathrm{~s}$, and a green (or blue) bead was associated with no-competition and 3 grains after $\Delta=0 \mathrm{~s}$. We had confirmed that chicks distinguished these two colors in experiment 1. Chicks learned this association from day 7 to day 9 , and were tested between two colors (day 10). In the second set of blocks, similarly, colored beads were associated with food both after $\Delta=1.5 \mathrm{~s}$. Chicks relearned from day 11 to day 13), and were tested (day 14). The color assignment counterbalanced in the group ( $n=3$ for each). (B) Numbers of choices of the colored bead associated with competition were recorded in the first and the second sets. Connected pair of circles denote each individual chick, and short horizontal bars indicate the median of the group.

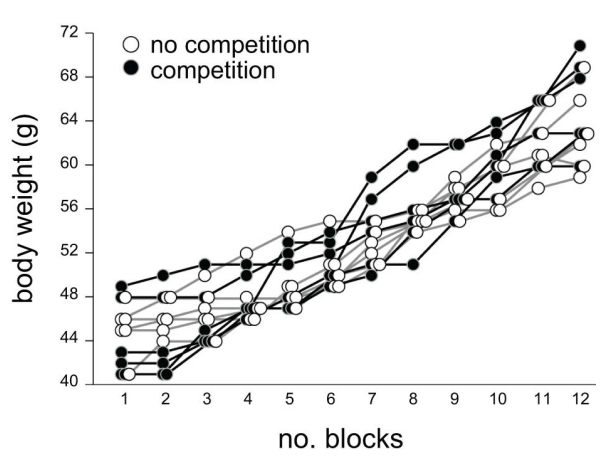

FIGURE A3 | Body weight of individuals in the two groups of chicks in experiment 2 (Figure 2) was plotted against number of blocks. Chicks of both groups similarly grew irrespectively of the training contexts. 